\title{
Distortion in transformation groups
}

\author{
DANNY CALEGARI \\ MICHAEL H FREEDMAN \\ APPENDIX BY YVES DE CORNULIER
}

\begin{abstract}
We exhibit rigid rotations of spheres as distortion elements in groups of diffeomorphisms, thereby answering a question of J Franks and $\mathrm{M}$ Handel. We also show that every homeomorphism of a sphere is, in a suitable sense, as distorted as possible in the group Homeo $\left(\mathbf{S}^{n}\right)$, thought of as a discrete group.

An appendix by $\mathrm{Y}$ de Cornulier shows that $\operatorname{Homeo}\left(\mathbf{S}^{n}\right)$ has the strong boundedness property, recently introduced by G Bergman. This means that every action of the discrete group Homeo $\left(\mathbf{S}^{n}\right)$ on a metric space by isometries has bounded orbits.
\end{abstract}

37C $85 ; 37 \mathrm{C} 05,22 \mathrm{~F} 05,57 \mathrm{~S} 25,57 \mathrm{M} 60$

\section{Introduction}

The study of abstract groups as geometric objects has a long history, but has been pursued especially vigorously since the work of Gromov [7;8]. Typically the focus is on finitely presented groups; however, interesting results have also been obtained from this perspective in the theory of transformation groups — ie groups of homeomorphisms of manifolds.

The topic of this paper is distortion in transformation groups, especially groups of homeomorphisms of spheres. Informally, an element $h$ in a finitely generated group $G$ is distorted if the word length of $h^{n}$ grows sublinearly in $n$. One also sometimes says that the translation length of $h$ vanishes. Geometrically, this corresponds to the condition that the homomorphism from $\mathbb{Z}$ to $G$ sending $n$ to $h^{n}$ is not a quasi-isometric embedding.

One can also make sense of the concept of distortion in infinitely generated groups. An element $h$ in a (not necessarily finitely generated) group $G$ is distorted if there is a finitely generated subgroup $H$ of $G$ containing $h$ such that $h$ is distorted in $H$ as above. To show that an element is undistorted, one typically tries to define an appropriate real-valued function on $G$ which is (almost) subadditive, and which grows linearly on $h^{n}$. For example, quasi-morphisms are useful in this respect, and highlight 
one point of contact between distortion and the theory of bounded cohomology. On the other hand, exhibiting distortion is typically done $a d$ hoc, and there do not seem to be many very general or flexible constructions known.

In this paper, we study distortion in groups of homeomorphisms of spheres, especially groups consisting of transformations with a definite amount of analytic regularity (ie $C^{1}$ or $C^{\infty}$ ). By contrast with Polterovich [19], Franks-Handel [4] or Ghys-Gambaudo [6], we do not insist that our groups preserve a probability measure; the considerable additional flexibility this affords has the consequence that our results have more of an existential character than those of the papers cited above, exhibiting distortion rather than ruling it out.

\subsection{Statement of results}

Notation 1.1 The letters $G, H$ will denote groups of some sort, and $S$ a (symmetric) generating set, although $\mathbf{S}^{n}$ denotes the $n$-sphere. If $G$ is a group, and $H$ is a subgroup, we write $H<G$. The group $G$ will often be a transformation group on some manifold, and a typical element $h$ will be a homeomorphism or diffeomorphism of some analytic quality. The letters $i, j, n$ will denote integers, $r$ will denote a degree of smoothness, and $g$ will denote a growth function (ie a function $g: \mathbb{N} \rightarrow \mathbb{N}$ ). $c$ and $k$ will usually denote (implicit) constants in some inequality. We let $\mathbb{R}_{+}$denote the non-negative real numbers. Other notation will be introduced as needed.

In Section 2 and Section 3 we summarize some basic definitions and study examples of distorted and undistorted elements in various groups.

In Section 4 we exhibit rigid rotations of $\mathbf{S}^{2}$ as distortion elements in the group of $C^{\infty}$ diffeomorphisms of the sphere.

Our main result in this section is:

Theorem $\mathbf{A}$ For any angle $\theta \in[0,2 \pi)$ the rigid rotation $R_{\theta}$ of $\mathbf{S}^{2}$ is a distortion element in a finitely generated subgroup of Diff ${ }^{\infty}\left(\mathbf{S}^{2}\right)$. Moreover, the distortion function of $R_{\theta}$ can be chosen to grow faster than any given function.

Here $R_{\theta}$ is a clockwise rotation about a fixed axis through angle $\theta$. To say that the distortion function grows faster than any given function means that for any $g: \mathbb{N} \rightarrow \mathbb{N}$ we can find a finitely generated group $G<\operatorname{Diff}^{\infty}\left(\mathbf{S}^{2}\right)$ for which there are words of length $\sim n_{i}$ in the generating set which express powers $R_{\theta}^{f\left(n_{i}\right)}$ of $R_{\theta}$ for some sequence $n_{i} \rightarrow \infty$, where $f(n)>g(n)$ for all sufficiently large $n \in \mathbb{N}$. In this case we say that the distortion function grows faster than $g$. 
This answers a question of John Franks and Michael Handel, motivated by results in their paper [4].

In Section 5 we go down a dimension, and study rigid rotations of $\mathbf{S}^{1}$. Our main result here is:

Theorem $\mathbf{B}$ For any angle $\theta \in[0,2 \pi)$ the rigid rotation $R_{\theta}$ of $\mathbf{S}^{1}$ is a distortion element in a finitely generated subgroup of $\operatorname{Diff}^{1}\left(\mathbf{S}^{1}\right)$. Moreover, the distortion function of $R_{\theta}$ can be chosen to grow faster than any given function.

The proof of Theorem B makes use of Pixton's results from [17], and the arguments should be familiar to people working in the theory of foliations. It should be remarked that our construction cannot be made $C^{2}$, and it appears to be unknown whether a rigid rotation of $\mathbf{S}^{1}$ is distorted in $\operatorname{Diff}{ }^{\infty}\left(\mathbf{S}^{1}\right)$ (or even in $\operatorname{Diff}^{2}\left(\mathbf{S}^{1}\right)$ ).

Remark 1.2 The possibility of proving Theorem B was pointed out to the first author by Franks and Handel, after reading an early version of this paper.

In Section 6 we relax our analytic conditions completely, and study distortion in the full group of homeomorphisms of $\mathbf{S}^{n}$. Here our main result is quite general:

Theorem C Fix $n \geq 1$. Let $h_{1}, h_{2}, \ldots$ be any countable subset of Homeo( $\left.\mathbf{S}^{n}\right)$, and $g_{1}, g_{2}, \ldots: \mathbb{N} \rightarrow \mathbb{N}$ any countable collection of growth functions. Then there is a finitely generated subgroup $H$ of $\operatorname{Homeo}\left(\mathbf{S}^{n}\right)$ (depending on $\left\{h_{i}\right\}$ and $\left\{g_{i}\right\}$ ) such that every $h_{i}$ is simultaneously distorted in $H$. Moreover, the distortion function of $h_{i}$ grows faster than $g_{i}$.

The proof of Theorem $\mathrm{C}$ uses the full power of the Kirby-Siebenmann theory of homeomorphisms of manifolds for a key factorization step. It is an interesting question whether one can exhibit distortion in an arbitrary homeomorphism of the sphere without recourse to such sophisticated technology.

Finally, in an appendix, Yves de Cornulier uses the proof of Theorem $\mathrm{C}$ to show that the group Homeo $\left(\mathbf{S}^{n}\right)$ is strongly bounded. Here an abstract group $G$ is said to be strongly bounded if every symmetric subadditive non-negative real-valued function on $G$ is bounded. A countable group has this property if and only if it is finite.

\subsection{Acknowledgements}

The first author would like to thank Michael Handel for suggesting the problem which motivated Theorem A, and to thank him and John Franks for reading preliminary versions of this paper, and for making clarifications and corrections. He would also like to thank Daniel Allcock for some useful comments. 


\section{Distortion elements}

\subsection{Conjugation notation}

Notation 2.1 For a group $G$ and elements $a, b \in G$, we abbreviate the conjugate $b^{-1} a b$ by

$$
a^{b}:=b^{-1} a b
$$

Notice with this convention that

$$
\left(a^{b}\right)^{c}=a^{b c} .
$$

\subsection{Basic definitions}

Definition 2.2 Let $G$ be a finitely generated group, and let $S$ be a finite generating set. By convention, we assume $S=S^{-1}$. Given $h \in G$, the length of $h$ with respect to $S$ is the minimum integer $n$ such that $h$ can be expressed as a product

$$
h=s_{1} s_{2} \cdots s_{n}
$$

where each $s_{i} \in S$. We write

$$
\ell_{S}(h)=n \text {. }
$$

By convention, we take $\ell_{S}(1)=0$.

Note that $\ell_{S}$ is a subadditive function; that is, for all $h_{1}, h_{2} \in G$,

$$
\ell_{S}\left(h_{1} h_{2}\right) \leq \ell_{S}\left(h_{1}\right)+\ell_{S}\left(h_{2}\right) .
$$

Moreover, it is non-negative and symmetric; ie $\ell(h)=\ell\left(h^{-1}\right)$. This motivates the definition of a length function on a group $G$.

Definition 2.3 Let $G$ be a group. A length function on $G$ is a function $L: G \rightarrow \mathbb{R}_{+}$ satisfying $L(1)=0$ which is symmetric and subadditive.

The function $\ell_{S}$ depends on the choice of generating set $S$, but only up to a multiplicative constant:

Lemma 2.4 If $S_{1}, S_{2}$ are two finite generating sets for $G$, then there is a constant $c \geq 1$ such that

$$
\frac{1}{c} \ell_{S_{2}}(h) \leq \ell_{S_{1}}(h) \leq c \ell_{S_{2}}(h)
$$

for all $h \in G$. 
Proof Each $s \in S_{1}$ can be expressed as a word of length $n(s)$ in the elements of $S_{2}$, and vice versa. Then take $c$ to be the maximum of the $n(s)$ over all $s \in S_{1} \cup S_{2}$.

Definition 2.5 Let $G$ be a finitely generated group, and let $S$ be a symmetric finite generating set as above. The translation length of an element $h \in G$, denoted $\|h\|_{S}$, is the limit

$$
\|h\|_{S}:=\lim _{n \rightarrow \infty} \frac{\ell_{S}\left(h^{n}\right)}{n} .
$$

An element $h \in G$ is a distortion element if the translation length is 0 .

Remark 2.6 Note that by the subadditivity property of $\ell_{S}$, the limit exists. Moreover, by Lemma 2.4 , the property of being a distortion element is independent of the choice of generating set $S$.

Remark 2.7 With this definition, torsion elements are distortion elements. Some authors (including Franks and Handel [4]) explicitly require distortion elements to be nontorsion.

Sometimes, we shall pay attention to the growth rate of $\ell_{S}\left(h^{n}\right)$ as a function of $n$ to make qualitative distinctions between different kinds of distortion elements. If $h$ is not torsion, we define the distortion function to be the function

$$
D_{S, h}: \mathbb{N} \rightarrow \mathbb{N}
$$

defined by the property

$$
D_{S, h}(n)=\max \left\{i \mid \ell_{S}\left(h^{i}\right) \leq n\right\} .
$$

We can remove the dependence of this function on $S$ as follows. For two functions

$$
f, g: \mathbb{N} \rightarrow \mathbb{N}
$$

we write $f \precsim g$ if there is a constant $k \geq 1$ such that

$$
f(n) \leq k g(k n+k)+k \text { for all } n \in \mathbb{N}
$$

and then write $f \sim g$ if $f \precsim g$ and $g \precsim f$. It is straightforward to see that $\precsim$ is transitive, and that $\sim$ is an equivalence relation. In case $f \sim g$, we say that $f, g$ are quasi-equivalent. With this definition, the quasi-equivalence class of $D_{S, h}$ is independent of $S$, and may be denoted $D_{h}$.

We are also interested in comparing growth rates in a cofinal sense: 
Definition 2.8 Given $g: \mathbb{N} \rightarrow \mathbb{N}$ we say that the distortion function of $h \in G$ (with respect to a generating set $S$ ) grows faster than $g$ if there is a sequence $n_{i} \rightarrow \infty$ and a function $f: \mathbb{N} \rightarrow \mathbb{N}$ such that $f(n)>g(n)$ for all sufficiently large $n$, and such that

$$
\ell_{S}\left(h^{f\left(n_{i}\right)}\right) \leq n_{i}
$$

We say for example that $h$ has quadratic distortion if $g(n)=n^{2}$ or exponential distortion if $g(n)=e^{n}$ as above.

Finally, we may define a distortion element in an arbitrary group:

Definition 2.9 Let $G$ be a group. An element $h \in G$ is a distortion element if there is a finitely generated subgroup $H<G$ with $h \in H$ such that $h$ is a distortion element in $H$.

Note that for such an element $h$, the quasi-equivalence class of the distortion function may certainly depend on $H$.

\subsection{Examples}

Example 2.10 In $\mathbb{Z}$ only the identity element is distorted.

Example 2.11 If $\phi: G \rightarrow H$ is a homomorphism, and $\phi(h)$ is not distorted in $H$, then $h$ is not distorted in $G$.

Example 2.12 If $L: G \rightarrow \mathbb{R}_{+}$is a length function, and

$$
\lim _{n \rightarrow \infty} \frac{L\left(h^{n}\right)}{n}>0
$$

then $h$ is not distorted. More generally, a length function gives a lower bound for word length with respect to any finite generating set, and therefore an upper bound on distortion. For example, if $L\left(h^{n}\right)$ grows like $\log (n)$ then $h$ is no more than exponentially distorted.

The next few examples treat distortion in linear groups.

Example 2.13 Let $G=\mathrm{GL}(n, \mathbb{C})$ and define $L: G \rightarrow \mathbb{R}_{+}$by

$$
L(A)=\log \text { of the max of the operator norms of } A \text { and } A^{-1} .
$$

Then $L$ is a length function. It follows that if $A$ has an eigenvalue with absolute value $\neq 1$ then $A$ is not distorted. 
Example 2.14 Let $\sigma \in \operatorname{Gal}(\mathbb{C} / \mathbb{Q})$ be a Galois automorphism of $\mathbb{C}$. Then $A$ is distorted in $\operatorname{GL}(n, \mathbb{C})$ if and only if $\sigma(A)$ is. It follows that if $A$ is distorted, then every eigenvalue must be algebraic, with all conjugates on the unit circle.

Example 2.15 Let $G<\mathrm{GL}(n, \mathbb{C})$ be a finitely generated subgroup with entries in a number field $K$. We may construct length functions from valuations associated to finite primes in the ring of integers of $K$. If $x \in K$ then $v(x)=0$ for all discrete valuations $v$ on $K$ if and only if $x$ is a unit. A unit in a number field with absolute value 1 is a root of unity; cf Lang [13]. Combined with Example 2.13 and Example 2.14 , one can show that an arbitrary element $A \in \mathrm{GL}(n, \mathbb{C})$ is distorted if and only if every eigenvalue of $A$ is a root of unity. Note that the distortion of a non-torsion element is at most exponential. See Lubotzky-Mozes-Raghunathan [14] for details.

Example 2.16 In the Baumslag-Solitar group $\left\langle a, b \mid a b a^{-1}=b^{2}\right\rangle$ the element $b$ has exponential distortion. Similarly, in the group

$$
\left\langle a, b, c \mid a b a^{-1}=b^{2}, b c b^{-1}=c^{2}\right\rangle
$$

the element $c$ has doubly-exponential distortion. Note that as a corollary, we deduce that this second group is not linear. This example and others are mentioned by Gromov $[8$, Chapter 3].

Example 2.17 Let $G$ be a group. A quasi-morphism is a map $\phi: G \rightarrow \mathbb{R}$ such that there is a constant $c>0$ for which

$$
\left|\phi\left(h_{1}\right)+\phi\left(h_{2}\right)-\phi\left(h_{1} h_{2}\right)\right| \leq c
$$

for all $h_{1}, h_{2} \in G$. If $|\phi(h)|>c$ then $h$ is not distorted.

Quasi-morphisms are intimately related to (second) bounded cohomology. See eg Ghys-Gambaudo [6] for a salient discussion.

\section{Distortion in transformation groups}

\subsection{Transformation groups}

Notation 3.1 For a compact $C^{\infty}$ manifold $M$, we denote the group of homeomorphisms of $M$ by Homeo $(M)$, and the group of $C^{r}$ diffeomorphisms by $\operatorname{Diff}^{r}(M)$, where $r=\infty$ is possible. Here a homeomorphism $h$ is in $\operatorname{Diff}^{r}(M)$ if both it and its inverse are $C^{r}$. Note that this implies $d h$ has full rank everywhere. If we wish to restrict to orientation-preserving subgroups, we denote this by a + superscript. 


\subsection{Distortion in Diff 1}

Suppose $M$ is a smooth compact Riemannian manifold, and $h \in \operatorname{Diff}^{1}(M)$. We define the following norm:

$$
\|d h\|:=\log \sup _{v \in U T M}|d h(v)|
$$

where $|d h(v)|$ denotes the length of $d h(v)$, and the supremum is taken over all vectors $v$ in the unit tangent bundle of $M$.

Note that since $h$ is a diffeomorphism and $M$ is compact, $d h$ cannot be strictly contracting at every point, and therefore $\|d h\| \geq 0$. If we define

$$
\|d h\|^{+}=\max \left(\|d h\|,\left\|d\left(h^{-1}\right)\right\|\right)
$$

then it is clear that $\|d \cdot\|^{+}$is a length function on $\operatorname{Diff}^{1}(M)$. In general, therefore, the growth rate of $\left\|d h^{n}\right\|^{+}$as a function of $n$ puts an upper bound on the distortion function of $h$ in any finitely generated subgroup of $\operatorname{Diff}^{1}(M)$.

Note if we choose two distinct Riemannian metrics on $M$, the length functions $\|d \cdot\|^{+}$ they define will be quasi-equivalent, by compactness. On the other hand, if $M$ is non-compact, different quasi-isometry classes of Riemannian metrics may give rise to qualitatively different length functions.

Example 3.2 Suppose $h$ has a fixed point $p$ and $\left.d h\right|_{T p M}$ has an eigenvalue with absolute value $\neq 1$. Then $h$ is not distorted in $\operatorname{Diff}^{1}(M)$.

Example 3.3 Oseledec's theorem (see Pollicott [18, Chapter 2]) says that for $h \in$ $\operatorname{Diff}^{1}(M)$ where $M$ is a compact manifold, and for $\mu$ an ergodic $h$-invariant probability measure on $M$, there are real numbers $\lambda_{1}>\cdots>\lambda_{k}$ called Lyapunov exponents, and a $\mu$-measurable $d h$-invariant splitting $T M=\oplus_{i=1}^{k} E^{i}$ such that

$$
\lim _{n \rightarrow \infty} \frac{1}{n} \log \left|d h^{n}(v)\right|=\lambda_{l}
$$

for almost every $v \in \oplus_{i=l}^{k} E^{i}$ but not in $\oplus_{i=l+1}^{k} E^{i}$. In particular, if $\lambda_{1}>0$, then $h$ is undistorted in $\operatorname{Diff}^{1}(M)$.

Example 3.4 Let $M$ be a compact manifold, and suppose $h \in \operatorname{Diff}^{1}(M)$ has positive topological entropy. Then there is an ergodic $h$-invariant probability measure $\mu$ for which $h$ has positive $\mu$-entropy. The Pesin-Ruelle inequality (see [18, Chapter 3]) says

$$
\sum_{\lambda_{i}>0} \lambda_{i} \geq \mu \text {-entropy of } h
$$


where the $\lambda_{i}$ are the Lyapunov exponents for $h$ with respect to the measure $\mu$. It follows that some Lyapunov exponent $\lambda_{1}$ for $\mu$ is positive, and therefore, as in Example 3.3, $h$ is undistorted in Diff ${ }^{1}(M)$.

By contrast, if $\left\|d h^{n}\right\|^{+}$is bounded independently of $n$, then the group $\langle h\rangle$ is equicontinuous, and is precompact in the group of Lipschitz homeomorphisms of $M$, by the Arzela-Ascoli theorem. By Repovš-Ščepin [21] (ie the Hilbert-Smith conjecture for Lipschitz actions), a compact group of Lipschitz homeomorphisms of a smooth manifold $M$ is a Lie group. In our case, this group is abelian, since it contains a dense abelian subgroup $\langle h\rangle$, and is therefore (up to finite index) a finite dimensional torus. Thus the uniformly equicontinuous case reduces to that of torus actions.

A key case to understand in this context is when the torus in question is $\mathbf{S}^{1}$, and the simplest example is that of a rigid rotation of a sphere. It is this example which we study in the next few sections.

\section{Rotations of $S^{2}$}

\subsection{The group $G$}

We describe a particular explicit group $G<\operatorname{Diff}{ }^{\infty}\left(\mathbf{S}^{2}\right)$ which will be important in the sequel. By stereographic projection, we may identify $\mathbf{S}^{2}$ conformally with $\mathbb{C} \cup \infty$.

Let $T$ be the similarity

$$
T: z \rightarrow 2 z \text {. }
$$

Then $\langle T\rangle$ acts discretely and properly discontinuously on $\mathbb{C}^{*}$ with quotient a (topological) torus. A fundamental domain for the action is the annulus $A$ defined by

$$
A=\{z \in \mathbb{C}|1 \leq| z \mid \leq 2\} .
$$

We let $\partial A^{+}$and $\partial A^{-}$denote the components $|z|=2$ and $|z|=1$ of $\partial A$ respectively. We define a disk $D$ by

$$
D=\{z \in \mathbb{C}|| z-3 / 2 \mid \leq 1 / 4\} .
$$

We let $F$ be a $C^{\infty}$ diffeomorphism with the following properties:

- $F$ is the identity outside the annulus $0.99 \leq|z| \leq 2.01$

- $F$ restricted to the annulus $1.01 \leq|z| \leq 1.99$ agrees with the rotation $z \rightarrow-z$ 
We define $G=\langle T, F\rangle$, and think of it as a subgroup of Diff ${ }^{\infty}\left(\mathbf{S}^{2}\right)$ fixing 0 and $\infty$. Notice that for every $h \in G$ either $h(D)$ is disjoint from $D$, or else $\left.h\right|_{D}=\left.\mathrm{Id}\right|_{D}$. If $G^{D}$ denotes the stabilizer of $D$ in $G$, then we may identify the orbit $G D$ with the product $D \times S$ where $S$ is the set of (right) cosets of the subgroup $G^{D}$ in $G$. Note that $S$ is a set with a (left) $G$-action. This action determines the action of $G$ on $D \times S$.

An explicit set of coset representatives for $S$ is the set of elements of the form $T^{n}$ and $F T^{n}$ for all $n \in \mathbb{Z}$.

\subsection{Wreath products}

Let $G, S$ and $D \subset \mathbf{S}^{2}$ be as in Section 4.1. Let $\zeta_{t}, t \in \mathbb{R}$ be a 1-parameter subgroup of diffeomorphisms of the unit disk with support contained in the interior. After conjugating by a diffeomorphism, we think of $\zeta_{t}$ as a 1-parameter subgroup of Diff ${ }^{\infty}\left(\mathbf{S}^{2}\right)$ with support contained in the interior of $D$.

Definition 4.1 Let $\mathbb{R}^{S}$ denote the set of functions from $S$ to $\mathbb{R}$, which can be thought of as an abelian group with respect to addition. The wreath product $G \imath_{S} \mathbb{R}$ is the semi-direct product

$$
0 \rightarrow \mathbb{R}^{S} \rightarrow G \imath_{S} \mathbb{R} \rightarrow G \rightarrow 0
$$

where $G$ acts on $\mathbb{R}^{S}$ by

$$
f^{h}(s)=f(h s)
$$

for $h \in G, s \in S$.

The choice of 1-parameter group $\zeta_{t}$ determines a faithful homomorphism

$$
\rho: G \imath_{S} \mathbb{R} \rightarrow \operatorname{Homeo}\left(\mathbf{S}^{2}\right)
$$

as follows. For $f \in \mathbb{R}^{S}$, define

$$
\rho(f)=\prod_{s \in S} \zeta_{f(s)}^{\bar{s}}
$$

where $\bar{s} \in G$ is a coset representative of $s \in S$. Together with the action of $G$ on $\mathbf{S}^{2}$ (in its capacity as a transformation group) this defines a faithful homomorphism $\rho$. For the sake of brevity, in the sequel we will omit $\rho$, and think of $G$ ?S $\mathbb{R}$ itself as a subgroup of Homeo( $\left.\mathbf{S}^{2}\right)$. 


\subsection{Analytic quality}

Given $f \in \mathbb{R}^{S}$, thought of as an element of Homeo( $\left(\mathbf{S}^{2}\right)$ as in Section 4.2, the analytic quality of $f$ is a priori only $C^{0}$. However, if we can estimate the $C^{r}$ norm of $f\left(T^{n}\right), f\left(F T^{n}\right)$ as $|n| \rightarrow \infty$, we can improve this a priori estimate.

Notice that any $f \in \mathbb{R}^{S}$ is $C^{\infty}$ away from $0, \infty$. In particular, any $f$ with finite support is $C^{\infty}$ on all of $\mathbf{S}^{2}$. Furthermore, conjugation by $T$ preserves the $C^{1}$ norm, and blows up the $C^{r}$ norm by $2^{r-1}$, whereas conjugation by $F$ preserves the $C^{r}$ norm for every $r$. It follows that if we have an estimate

$$
\left|f\left(T^{n}\right)\right|,\left|f\left(F T^{n}\right)\right|=o\left(2^{-|n|(r-1)}\right)
$$

as $|n| \rightarrow \infty$, then $f$ is $C^{r}$ at 0 (here our notation $|f(s)|$ just means the absolute value of $f(s)$ for $s \in S$, where we think of $f$ as a function from $S$ to $\mathbb{R})$. By the change of co-ordinates $z \rightarrow 1 / z$ one sees that $f$ is also $C^{r}$ at $\infty$ under the same hypothesis, and is therefore $C^{r}$ on all of $\mathbf{S}^{2}$.

We summarize this as a lemma:

Lemma 4.2 Let $f \in \mathbb{R}^{S}$ be thought of as an element of Homeo($\left(\mathbf{S}^{2}\right)$ as in Section 4.2. Then we have the following estimates:

- If $|f(s)|$ is bounded independently of $s \in S$ then $f$ is Lipschitz

- If $\lim _{s \rightarrow \infty}|f(s)|=0$ then $f$ is $C^{1}$

- If $\left|f\left(T^{n}\right)\right|,\left|f\left(F T^{n}\right)\right| \rightarrow 0$ faster than any exponential (as a function of $n$ ), then $f$ is $C^{\infty}$

\subsection{Rotations of $\mathrm{S}^{2}$}

For each $\theta \in[0,2 \pi)$ we let $R_{\theta}$ denote the rigid rotation of $\mathbf{S}^{2}$ with fixed points equal to 0 and $\infty$. In stereographic co-ordinates,

$$
R_{\theta}: z \rightarrow e^{i \theta} z
$$

where $z \in \mathbb{C} \cup \infty$. Notice that $R_{\pi}$ is just multiplication by -1 .

For $\theta \in \pi \mathbb{Q}$ the element $R_{\theta}$ is torsion in $\operatorname{Diff}^{\infty}\left(\mathbf{S}^{2}\right)$. We will show in this section that $R_{\theta}$ is a distortion element in $\operatorname{Diff}^{\infty}\left(\mathbf{S}^{2}\right)$ for arbitrary $\theta$. Moreover, the distortion function can be taken to grow faster than any given function. 


\subsection{Factorizing rotations}

We can factorize $R_{\theta}$ in a natural way as a product of two diffeomorphisms whose support is contained in closed subdisks of $\mathbf{S}^{2}$. This will be important for some later applications.

Let $B$ (for bump) be a smooth function $B: \mathbb{R}^{+} \rightarrow[0,1]$ which satisfies the following properties:

- $B(t)=0$ for $t<1 / 2$ and $B(t)=1$ for $t>2$

- $B(t)+B(1 / t)=1$

- $B$ is monotone decreasing and strictly positive on $(1 / 2,2)$

- $B$ is infinitely tangent to the constant function 1 at 2 and to the constant function 0 at $1 / 2$

For $\theta \in \mathbb{R}$, define $R_{\theta}^{+}$by

$$
R_{\theta}^{+}: z \rightarrow e^{i B(|z|) \theta} z
$$

and define $R_{\theta}^{-}$by the identity

$$
R_{\theta}^{+} R_{\theta}^{-}=R_{\theta} .
$$

Notice that as $\theta$ varies over $\mathbb{R}$, the set of transformations $R_{\theta}^{-}$and $R_{\theta}^{+}$form smooth subgroups of $\operatorname{Diff}^{+}\left(\mathbf{S}^{2}\right)$. Moreover, the support of the group $\left\{R_{\theta}^{-} \mid \theta \in \mathbb{R}\right\}$ is equal to the disk

$$
E^{-}=\{z|| z \mid \leq 2\}
$$

Similarly, the support of $R_{\theta}^{+}$is the disk (in $\mathbf{S}^{2}$ )

$$
E^{+}=\{z|| z \mid \geq 1 / 2\} .
$$

Notice the important fact that $z \rightarrow 1 / z$ conjugates $R_{-\theta}^{+}$to $R_{\theta}^{-}$for any $\theta$. The reason for the sign change is that a 1-parameter family of rotations which has a clockwise sense at one fixed point has an anticlockwise sense at the other fixed point.

\subsection{Construction of the group}

Throughout the remainder of this section we assume that $\theta$ has been fixed.

We define a diffeomorphism $Z$ which takes care of some bookkeeping for us. Basically, the diffeomorphism $Z$ lets us move back and forth between the 1-parameter groups $R_{t}^{ \pm}$with support in $E^{ \pm}$and a 1-parameter group $\zeta_{t}$ with support in $D$, as in Section 4.1 and Section 4.2. The exact details of how this is done are irrelevant, but we must make an explicit choice, which accounts for the (annoying) notational complexity below.

Let $Z \in \operatorname{Diff}^{\infty}\left(\mathbf{S}^{2}\right)$ satisfy the following properties: 
- $Z$ takes $D$ to $E^{-}$and conjugates $R_{t}^{-}$to a 1 -parameter subgroup $\zeta_{t}$ :

$$
\zeta_{t}:=\left(R_{t}^{-}\right)^{Z}
$$

- $Z$ takes $F D$ to $T^{3} E^{+}$(ie the image of the disk $E^{+}$under the similarity $z \rightarrow 8 z)$ and conjugates $\left(R_{t}^{+}\right)^{T^{-3}}$ to $\zeta_{-t}^{F}$ :

$$
\zeta_{-t}^{F}=\left(R_{t}^{+}\right)^{T^{-3} Z}
$$

The existence of such a diffeomorphism $Z$ follows from the disjointness of the disks $E^{-}, T^{3} E^{+}$and the fact that the subgroups $R_{t}^{-}$and $R_{-t}^{+}$are abstractly conjugate, by $z \rightarrow 1 / z$, as pointed out in Section 4.5.

Now form the group $\mathbb{R}^{S}$ as in Section 4.2 by means of the subgroup $\zeta_{t}=\left(R_{t}^{-}\right)^{Z}$.

Let $t_{i} \in \mathbb{R}$ be chosen for all non-negative integers $i$ subject to the following constraints:

- $t_{i}=n_{i} \theta \bmod 2 \pi$ where $n_{i} \rightarrow \infty$ grow as fast as desired (ie faster than some growth function $g: \mathbb{N} \rightarrow \mathbb{N}$ we are given in advance)

- $t_{i} \rightarrow 0$ faster than any exponential function

Define the element $f \in \mathbb{R}^{S}$ by

$$
f\left(T^{i}\right)=t_{i} \text { if } i \geq 0, f\left(T^{i}\right)=0 \text { if } i<0, f\left(F T^{i}\right)=0 \text { for all } i .
$$

By Lemma 4.2 the function $f$ is in Diff ${ }^{\infty}\left(\mathbf{S}^{2}\right)$ with respect to the identification of $\mathbb{R}^{S}$ with a subgroup of Homeo $\left(\mathbf{S}^{2}\right)$.

Now, for any $i$, the element

$$
f_{i}:=f^{T^{i}}\left(f^{T^{i} F}\right)^{-1}
$$

is contained in $\mathbb{R}^{S}$, and satisfies

$$
f_{i}(s)= \begin{cases}t_{i} & \text { if } s=\mathrm{Id} \\ -t_{i} & \text { if } s=F \\ 0 & \text { otherwise }\end{cases}
$$

We conjugate the $f_{i}$ back by $Z^{-1}$, and define

$$
h_{i}:=f_{i}^{Z^{-1}} \text {. }
$$

Then $h_{i}$ agrees with $R_{t_{i}}^{-}$on $E^{-}$and agrees with $\left(R_{t_{i}}^{+}\right)^{T^{-3}}$ on $T^{3} E^{+}$.

Notice that $h_{i}$ preserves the foliation of $\mathbf{S}^{2} \backslash\{0, \infty\}$ by circles of equal latitude, and acts on each of these circles by a rotation. Let LAT $<\operatorname{Diff}{ }^{\infty}\left(\mathbf{S}^{2}\right)$ denote the group of 
diffeomorphisms with this property; ie informally, LAT preserves latitude, and acts as a rotation on each circle with a fixed latitude. An element of LAT can be thought of as a $C^{\infty}$ function

$$
\text { latitude } \rightarrow \text { rotation angle }
$$

up to constant functions with values in $2 \pi \mathbb{Z}$, and any element of LAT can be recovered pictorially from the graph of this function. Notice that $h_{i} \in$ LAT. In this way, we can abbreviate $h_{i}$ by a picture:

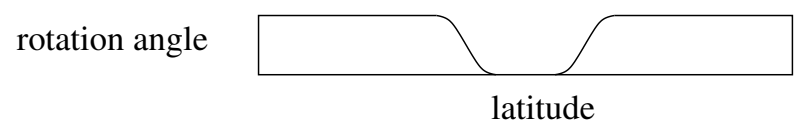

Figure 1: The element $h_{i} \in \mathrm{LAT}$ represented pictorially by the graph of a function Let $\mathrm{LONG}<\operatorname{Diff}{ }^{\infty}\left(\mathbf{S}^{2}\right)$ denote the group of diffeomorphisms of the form

$$
z \rightarrow z \cdot u(|z|)
$$

where $u: \mathbb{R}^{+} \rightarrow \mathbb{R}^{+}$is infinitely tangent to the identity at 0 and at $\infty$. Informally, LONG is the group of diffeomorphisms which reparameterizes the set of latitudes, without changing longitudes. Then LONG is contained in the normalizer of the group LAT. The conjugation action of LONG on LAT is given pictorially by reparameterizing the base of the graph.

We claim that there are elements $M_{1}, M_{2}, M_{3} \in$ LONG such that for any $h_{i}$ we have an identity

$$
h_{i}\left(h_{i}\right)^{M_{1}}\left(h_{i}\right)^{M_{2}}\left(\left(h_{i}\right)^{M_{3}}\right)^{-1}=R_{2 t_{i}} .
$$

The proof is given graphically by Figure 2 :

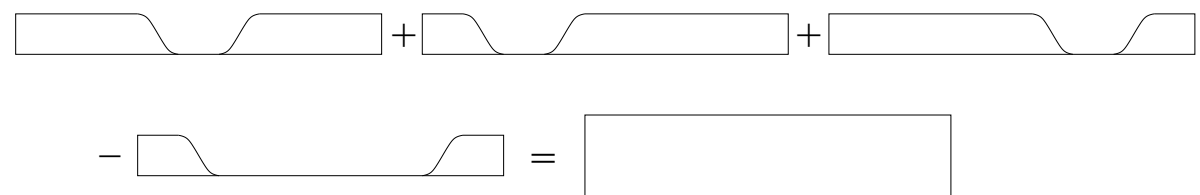

Figure 2: These figures denote the conjugates of $h_{i}$, and demonstrate how an appropriate algebraic product of these conjugates is equal to $R_{2 t_{i}}$

Now, $R_{2 t_{i}}=R_{\theta}^{2 n_{i}}$. Since the $n_{i}$ have been chosen to grow faster than any function given in advance, we have proved the following theorem:

Theorem $\mathbf{A}$ For any angle $\theta \in[0,2 \pi)$ the rigid rotation $R_{\theta}$ of $\mathbf{S}^{2}$ is a distortion element in a finitely generated subgroup of $\operatorname{Diff}{ }^{\infty}\left(\mathbf{S}^{2}\right)$. Moreover, the distortion function of $R_{\theta}$ can be chosen to grow faster than any given function. 


\section{Rotations of $\mathrm{S}^{1}$}

In this section we show how to modify the construction of Section 4 to exhibit a rigid rotation as a distortion element in the group $\operatorname{Diff}^{1}\left(\mathbf{S}^{1}\right)$. But first, we exhibit a rotation as a distortion element in the group of Lipschitz homeomorphisms of $\mathbf{S}^{1}$.

\subsection{Rotations of $\mathrm{S}^{1}$}

As in the previous section, we denote by $R_{\theta}$ the rotation of $\mathbf{S}^{1}$ through angle $\theta \in$ $[0,2 \pi)$.

The first difference with Section 4 is that we cannot factorize a 1-parameter group of rotations as the product of two 1-parameter groups with support contained in an interval. (One way to see this is to use Poincaré's rotation number; see eg Sinai [24] for a definition and basic properties.)

Let $\theta$ be fixed, and we choose $t_{i} \rightarrow 0, n_{i} \rightarrow \infty$ as $i \in \mathbb{Z}$ goes from 0 to $\infty$, with

$$
t_{i}=n_{i} \theta \bmod 2 \pi
$$

as in Section 4.6.

Let $I^{ \pm}$be two intervals which form an open cover of $\mathbf{S}^{1}$. Then for $t_{i}$ sufficiently close to 0 , we can factorize $R_{t_{i}}$ as a product of two diffeomorphisms $\xi_{i}, \zeta_{i}$ with support contained in $I^{+}, I^{-}$respectively. It is clear that we may choose $\xi_{i}, \zeta_{i}$ so that their support is exactly equal to an interval, and they are both conjugate to translations on these intervals.

Let $J$ be an open interval in $\mathbf{S}^{1}$ which we parameterize by arclength as $[-1,1]$. We let $T$ be a diffeomorphism of $\mathbf{S}^{1}$ with support equal to $J$, and with no fixed points in $J$. Then the restriction of $T$ to $J$ is conjugate to a translation, and we let $J_{i}$ for $i \in \mathbb{Z}$ be a tiling of $J$ by fundamental domains for $t$.

Fix one such interval $J_{0} \subset J$ and let $F$ be a diffeomorphism of $\mathbf{S}^{1}$ with support equal to $J_{0}$, and with no fixed points in $J_{0}$. We let $J_{0 i}$ for $i \in \mathbb{Z}$ be a tiling of $J_{0}$ by fundamental domains for $F$.

The group $G=\langle T, F\rangle$ acts as before on the set of translates of $J_{00}$, and for all $h \in G$, either $h\left(J_{00}\right)$ is disjoint from $J_{00}$, or else $\left.h\right|_{J_{00}}=\left.\mathrm{Id}\right|_{J_{00}}$. The interval $J_{00}$ is the analogue of the disk $D$ from Section 4, and the elements $T, F$ are the analogues of the diffeomorphisms of the same names in that section. The difference is that if $G J_{00}$ denotes the set of translates of $J_{00}$ by $G$, then $\left.F\right|_{G J_{00}}$ has infinite order, rather than order 2 . 
Now let $Z^{ \pm}$be diffeomorphisms of $\mathbf{S}^{1}$ taking $I^{ \pm}$respectively to the interval $J_{00}$. The diffeomorphisms $Z^{ \pm}$are the analogue of the diffeomorphism $Z$ from Section 4; the reason we need two such diffeomorphisms rather than just one is that the factorization of $R_{t_{i}}$ into $\xi_{i} \zeta_{i}$ is no longer canonical.

We let $f^{+} \in \operatorname{Homeo}\left(\mathbf{S}^{1}\right)$ have support contained in $J$, and define it to be the product

$$
f^{+}=\prod_{i=0}^{\infty} \prod_{j=0}^{\infty} \xi_{i}^{\left(Z^{+}\right)^{-1} F^{-j} T^{-i}}
$$

and similarly, define

$$
f^{-}=\prod_{i=0}^{\infty} \prod_{j=0}^{\infty} \zeta_{i}^{\left(Z^{-}\right)^{-1} F^{-j} T^{-i}} .
$$

Notice by Lemma 4.2 that $f^{ \pm}$are Lipschitz (though not $C^{1}$ ).

Then for each $i$,

$$
\left(\left(f^{+}\right)^{T^{i}}\left(\left(f^{+}\right)^{T^{i} F^{-1}}\right)^{-1}\right)^{Z^{+}}=\xi_{i}
$$

and

$$
\left(\left(f^{-}\right)^{T^{i}}\left(\left(f^{+}\right)^{T^{i} F^{-1}}\right)^{-1}\right)^{Z^{-}}=\zeta_{i}
$$

and therefore $R_{n_{i} \theta}$ can be expressed as a word of length $\sim 8 i$ in the group

$$
\left\langle f^{+}, f^{-}, F, T, Z^{+}, Z^{-}\right\rangle \text {. }
$$

Notice that there is no analogue of the groups LAT and LONG, and consequently no analogue of the elements $M_{1}, M_{2}, M_{3}$.

\subsection{A $C^{1}$ example}

By a slight modification, using a trick of Pixton we can actually improve the Lipschitz example of Section 5.1 to a $C^{1}$ example.

We note that by suitable choice of factorization of $R_{t_{i}}$ we can assume the following:

- The support of $\xi_{i}, \zeta_{i}$ is exactly equal to $I^{+}, I^{-}$respectively

- On $I^{+}$, each $\xi_{i}$ is conjugate to a translation, and similarly for $I^{-}$

Now, the elements $\xi_{i}$ for distinct $i$ will not be contained in a fixed 1-parameter subgroup of Diff ${ }^{\infty}\left(I^{+}\right)$, but they are all conjugate into a fixed 1-parameter subgroup, and similarly for the $\zeta_{i}$. The final condition we insist on is: 
- The conjugating maps can be taken to be $C^{1}$ and converge in the $C^{1}$ topology to the identity.

To see that this is possible, observe that for two $C^{\infty}$ diffeomorphisms $\epsilon$-close to the identity in the $C^{1}$ norm, the commutator is $\epsilon^{2}$-close to the identity, also in the $C^{1}$ norm. So for diffeomorphisms $\phi_{g}$ defined by the property

$$
\phi_{g}: \theta \rightarrow \theta+g(\theta)
$$

for $g: \mathbf{S}^{1} \rightarrow \mathbb{R}$, we have that

$$
\phi_{g_{1}} \phi_{g_{2}} \sim \phi_{g_{1}+g_{2}}
$$

with error which is comparable in size in the $C^{1}$ norm to the products of the $C^{1}$ norms of $g_{1}, g_{2}$. Using this fact, one can readily produce a suitable factorization.

\subsection{Pixton actions}

Consider an interval $I$ on which a diffeomorphism $Y: I \rightarrow I$ acts in a manner smoothly conjugate to a translation, with fundamental domains $I_{i}$. Given another diffeomorphism $\phi: I_{0} \rightarrow I_{0}$ we form the suspension $\Phi: I \rightarrow I$ by

$$
\Phi=\prod_{i} \phi^{Y^{i}}
$$

Note that $\langle Y, \Phi\rangle \cong \mathbb{Z} \oplus \mathbb{Z}$. If $\phi$ restricted to $I_{0}$ is smoothly conjugate to a translation, then a priori the action of $\langle Y, \Phi\rangle$ on $I$ is Lipschitz. However, Pixton showed that it topologically conjugate (ie by a homeomorphism) to a $C^{1}$ action.

For the convenience of the reader, we give an outline of the construction of a Pixton action. One chooses co-ordinates on $I$ so that the ratio $\left|I_{i}\right| /\left|I_{i+1}\right|$ converges to 1 as $|i| \rightarrow \infty$. For instance, near $I$, the endpoints of the $I_{n}$ could be the harmonic series $1 / 2,1 / 3, \ldots$ so that the ratio of successive lengths is $i /(i+1) \rightarrow 1$. Then we require $Y: I_{i} \rightarrow I_{i+1}$ to expand the linear structure near the endpoints and contract it in the middle, so that the norm of the first derivative of $\left.\Phi\right|_{I_{i+1}}$ is smaller than that of $\left.\Phi\right|_{I_{i}}$ by a definite amount. Then both $Y$ and $\Phi$ are $C^{1}$ tangent to the identity at the endpoints of $I$, and are therefore $C^{1}$ on the entire interval. See Pixton [17] for rigorous details of this construction. One should remark that a lemma of Kopell [12] implies that one cannot make the action $C^{2}$.

This construction has the following virtue: if $\phi$ is contained in a smooth 1-parameter subgroup $\phi_{t}$, and we form the associated 1-parameter subgroup $\Phi_{t}$ so that

$$
\left\langle Y, \Phi_{t}\right\rangle \cong \mathbb{Z} \oplus \mathbb{R}
$$

then we can form a Pixton action of this larger group which is $C^{1}$. 


\section{$5.4 T$ and $X$}

Naively, one sees that by careful choice of $F$, one can arrange for the action of $\left.f^{+}\right|_{J_{0}}$ to be $C^{1}$. However, to make $f^{+} C^{1}$ on all of $J$ requires us to modify the definition slightly.

We will construct $X$, a diffeomorphism of $\mathbf{S}^{1}$ with support equal to $J$, conjugate to a translation on $J$, and with fundamental domains $J_{i}$, just like $T$.

We let $\chi_{t}$ be a 1 -parameter subgroup containing $\xi_{0}$. For each $i$, we require that

$$
\xi_{i}^{\left(Z^{+}\right)^{-1} T^{-i} X^{i}} \in\left(\chi_{t}\right)^{\left(Z^{+}\right)^{-1}}
$$

which is possible, by the discussion at the end of Section 5.2. By choosing co-ordinates on $J$ suitably as above, we can insist that both $X$ and $T$ are $C^{1}$.

Now we choose co-ordinates on $J_{0}$ so that $F$ and $\left(\chi_{t}\right)^{\left(Z^{+}\right)^{-1}}$ form a Pixton action of $\mathbb{Z} \oplus \mathbb{R}$ there, as in Section 5.3.

We define

$$
f^{+}=\prod_{i=0}^{\infty} \prod_{j=0}^{\infty} \xi_{i}^{\left(Z^{+}\right)^{-1} T^{-i} X^{i} F^{-j} X^{-i}} .
$$

Note that $f^{+}$is actually $C^{1}$.

Moreover, we have the following formula

$$
\left(\left(\left(f^{+}\right)^{X^{i}}\left(\left(f^{+}\right)^{X^{i} F^{-1}}\right)^{-1}\right)^{X^{-i} T^{i}}\right)^{Z^{+}}=\xi_{i} .
$$

Relabelling $X$ as $X^{+}$and defining $X^{-}$similarly in terms of the $\zeta_{i}$, one can define $f^{-}$analogously. Putting this together, we have shown

Theorem $\mathbf{B}$ For any angle $\theta \in[0,2 \pi)$ the rigid rotation $R_{\theta}$ of $\mathbf{S}^{1}$ is a distortion element in a finitely generated subgroup of $\operatorname{Diff}^{1}\left(\mathbf{S}^{1}\right)$. Moreover, the distortion function of $R_{\theta}$ can be chosen to grow faster than any given function.

One should remark that for a rigid rotation $R$ of $\mathbf{S}^{n}$ where $n$ is arbitrary, either $R$ has fixed points, in which case the construction of Section 4 shows that $R$ is a distortion element in Diff ${ }^{\infty}\left(\mathbf{S}^{n}\right)$, or else the construction of this section can be generalized to show that $R$ is a distortion element in $\operatorname{Diff}^{1}\left(\mathbf{S}^{n}\right)$, in either case with distortion growing faster than any given function. 
Remark 5.1 Tsuboi showed in [25] that one can construct Pixton actions which are $C^{1+\alpha}$ for every $\alpha<1$. It is therefore likely that the construction above exhibits a rigid rotation as an arbitrarily badly distorted element in $\operatorname{Diff}^{1+\alpha}\left(\mathbf{S}^{1}\right)$.

By our discussion in Section 3.2, we make the following conjecture:

Conjecture 5.2 Let $M$ be a compact smooth manifold, and let $h \in \operatorname{Diff}^{1}(M)$. Then $h$ is a distortion element in $\operatorname{Diff}^{1}(M)$ whose distortion function can be chosen to grow faster than any given function if and only if some finite power of $h$ is contained in a Lipschitz action of a finite dimensional torus on $M$.

Note that the "only if" direction follows from Section 3.2.

\section{Distortion in $\operatorname{Homeo}\left(\mathbf{S}^{n}\right)$}

The group Homeo $(M)$ for an arbitrary manifold $M$ is considerably more complicated than $\operatorname{Diff}^{\infty}(M)$ or even $\operatorname{Diff}^{1}(M)$. In this section, we first make a couple of comments about distortion in $\operatorname{Homeo}(M)$ in general, and then specialize to the case of Homeo $\left(\mathbf{S}^{n}\right)$.

\subsection{Mapping class groups}

For an arbitrary compact manifold $M$, there is a natural homomorphism

$$
\operatorname{Homeo}(M) \rightarrow \operatorname{Homeo}(M) / \operatorname{Homeo}_{0}(M)=: \operatorname{MCG}(M)
$$

where $\mathrm{Homeo}_{0}(M)$ is the normal subgroup consisting of homeomorphisms isotopic to the identity, and $\operatorname{MCG}(M)$ is the mapping class group of $M$. For reasonable $M$, this group is finitely presented, and quite amenable to computation. Clearly for $h \in \operatorname{Homeo}(M)$ to be a distortion element, it is necessary for the image $[h]$ of $h$ in $\operatorname{MCG}(M)$ to be a distortion element.

Example 6.1 A pseudo-Anosov homeomorphism of a closed surface $\Sigma$ of genus $\geq 2$ is not a distortion element in $\operatorname{Homeo}(\Sigma)$.

\subsection{Distortion in $\mathrm{Homeo}_{0}(M)$}

For suitable manifolds $M$, it is easy to find undistorted elements in $\operatorname{Homeo}_{0}(M)$. 
Example 6.2 Let $T^{2}$ denote the 2-torus. Let $h: T^{2} \rightarrow T^{2}$ preserve the foliation of $T^{2}$ by meridians, and act as a rigid rotation on each meridian, where the angle of rotation is not constant. This angle of rotation defines a map $\theta: \mathbf{S}^{1} \rightarrow \mathbf{S}^{1}$, where the first factor labels the meridian, and the second factor is the amount of rotation. If $\theta$ is homotopically trivial, $h$ is in $\operatorname{Homeo}_{0}\left(T^{2}\right)$. In this case, we claim $h$ is undistorted in Homeo $_{0}\left(T^{2}\right)$. To see this, suppose to the contrary that $h$ is distorted in some finitely generated subgroup $H$. Without loss of generality, we may expand $H$ to a larger finitely generated group, where each generator $h_{i}$ has support contained in a closed disk in $T^{2}$. If $\widetilde{h_{i}}$ denotes a lift of $h_{i}$ to the universal cover $\mathbb{R}^{2}$, then there is a constant $c$ such that

$$
\left|d_{\mathbb{R}^{2}}\left(\widetilde{h_{i}}(p), \widetilde{h_{i}}(q)\right)-d_{\mathbb{R}^{2}}(p, q)\right| \leq c
$$

for any $p, q \in \mathbb{R}^{2}$. Without loss of generality, we may assume that the same constant $c$ works for all $i$.

Now, if $I$ is a small transversal to the foliation of $T^{2}$ by meridians, intersecting meridians where the function $\theta$ is nonconstant, it follows that if we denote $I_{n}:=h^{n}(I)$, then a lift $\widetilde{I_{n}}$ of $I_{n}$ has the property that the endpoints are distance $\sim k n$ apart for some positive constant $k$. By the discussion above, this implies that any expression of $h^{n}$ in the generators $h_{i}$ and their inverses has word length at least $\sim n k / c$. This shows that $h$ is undistorted, as claimed.

Example 6.3 Let $M$ be a closed hyperbolic 3-manifold. Let $\gamma \subset M$ be a simple closed geodesic, and let $N$ be an embedded tubular neighborhood. Let $h: M \rightarrow M$ rotate $\gamma$ some distance, and be fixed outside $N$. Then the argument of Example 6.2 shows that $h$ is undistorted in $\operatorname{Homeo}_{0}(M)$. Since $M$ is hyperbolic of dimension at least 3, Mostow rigidity [15] implies that $\operatorname{MCG}(M)$ is finite. It follows that $h$ is undistorted in the full group $\operatorname{Homeo}(M)$.

Question 6.4 Is $h$ as in Example 6.2 undistorted in $\operatorname{Homeo}\left(T^{2}\right)$ ?

The method of construction in Example 6.2 produces an undistorted element of $\mathrm{Homeo}_{0}(M)$ when $\pi_{1}(M)$ contains an undistorted element. Moreover, if $\operatorname{MCG}(M)$ is finite, the element is undistorted in $\operatorname{Homeo}(M)$. This begs the following obvious question:

Question 6.5 Is there an infinite, finitely presented group $G$ in which every element is distorted?

Remark 6.6 A finitely presented infinite torsion group would answer Question 6.5 affirmatively. 
Remark 6.7 It is worth observing that Ol'shanskii [16] has shown the existence of a torsion-free finitely generated group in which all elements are distorted, thereby answering a question of Gromov.

The following construction gets around Question 6.5, at a mild cost.

Example 6.8 Let $M$ be a closed manifold with $\pi_{1}(M)$ infinite. Then $\widetilde{M}$ inherits a path metric pulled back from $M$ with respect to which the diameter is infinite. It follows that $\widetilde{M}$ contains a ray $r$ - that is, an isometrically embedded copy of $\mathbb{R}^{+}$ which realizes the minimal distance between any two points which it contains. The ray $r$ projects to $M$ where it might intersect itself. By abuse of notation, we refer to the projection as $r$. If the dimension of $M$ is at least 3, then we can perturb $r$ an arbitrarily small amount so that it is embedded in $M$ (though of course not properly embedded). In fact, we can even ensure that there is an embedded tubular neighborhood $N$ of $r$ whose width tapers off to zero as one escapes to infinity in $r$ in its intrinsic path metric. Let $h$ be a homeomorphism of $M$, fixed outside $N$, which translates the core (ie $r$ ) by some function

$$
r(t) \rightarrow r(t+f(t))
$$

where $f(t)$ is positive, and goes to 0 as $t \rightarrow \infty$. Such a homeomorphism may be constructed for instance by coning this translation of $r$ out to $\partial N$ with respect to some radial co-ordinates. Then $h$ might be distorted, but the distortion function can be taken to increase as slowly as desired, by making $f$ go to 0 as slowly as desired. For example, we could ensure that the distortion function grows slower than $n^{\alpha}$ for all $\alpha>1$.

\subsection{Homeomorphisms of spheres}

We now specialize to $\mathbf{S}^{n}$. We make use of the following seemingly innocuous lemma:

Lemma 6.9 (Kirby-Siebenmann, Quinn) Let $h \in \mathrm{Homeo}^{+}\left(\mathbf{S}^{n}\right)$. Then $h$ can be factorized as a product

$$
h=h_{1} h_{2}
$$

where the support of $h_{1}$ avoids the south pole, and the support of $h_{2}$ avoids the north pole.

For $h$ sufficiently close to the identity in the compact-open topology, this can be proved by the geometric torus trick. For an arbitrary homeomorphism, it requires the full 
power of topological surgery theory. See Kirby-Siebenmann [11] for details in the case $n \neq 4$ and Quinn [20] for the case $n=4$.

Using this lemma, we can produce another factorization:

Lemma 6.10 Let $E_{1}, E_{2}$ be two closed disks in $\mathbf{S}^{n}$ whose interiors cover $\mathbf{S}^{n}$. Then any $h \in \mathrm{Homeo}^{+}\left(\mathbf{S}^{n}\right)$ can be factorized as a product of at most 6 homeomorphisms, each of which has support contained in either $E_{1}$ or $E_{2}$.

Proof Without loss of generality, we can assume that $E_{1}$ and $E_{2}$ contain collar neighborhoods of the northern and southern hemisphere respectively.

Given $h \in \mathrm{Homeo}^{+}\left(\mathbf{S}^{n}\right)$, we factorize $h$ as $h_{1} h_{2}$ as in Lemma 6.9. Let $e_{2}$ be a radial expansion centered at the south pole, with support contained in $E_{2}$, which takes $\operatorname{supp}\left(h_{1}\right) \cap E_{2}$ into $E_{2} \cap E_{1}$. Then $e_{2} h_{1} e_{2}^{-1}$ has support contained in $E_{1}$. Similarly, we can find $e_{1}$ with support contained in $E_{1}$ such that $e_{1} h_{2} e_{1}^{-1}$ has support contained in $E_{2}$. Then

$$
h=e_{2}^{-1}\left(e_{2} h_{1} e_{2}^{-1}\right) e_{2} e_{1}^{-1}\left(e_{1} h_{2} e_{1}^{-1}\right) e_{1}
$$

expresses $h$ as the product of 6 homeomorphisms, each with support in either $E_{1}$ or $E_{2}$.

Remark 6.11 Notice in the factorization in Lemma 6.10 that the homeomorphisms $e_{1}, e_{2}$ definitely depend on $h$.

Theorem C Fix $n \geq 1$. Let $h_{1}, h_{2}, \ldots$ be any countable subset of Homeo( $\left.\mathbf{S}^{n}\right)$, and $g_{1}, g_{2}, \ldots: \mathbb{N} \rightarrow \mathbb{N}$ any countable collection of growth functions. Then there is a finitely generated subgroup $H$ of $\operatorname{Homeo}\left(\mathbf{S}^{n}\right)$ (depending on $\left\{h_{i}\right\}$ and $\left\{g_{i}\right\}$ ) such that every $h_{i}$ is simultaneously distorted in $H$. Moreover, the distortion function of $h_{i}$ grows faster than $g_{i}$.

Proof The subgroup Homeo ${ }^{+}\left(\mathbf{S}^{n}\right)$ of Homeo $\left(\mathbf{S}^{n}\right)$ has index 2, so after replacing each $h_{i}$ by $h_{i}^{2}$ if necessary, we can assume each $h_{i} \in \mathrm{Homeo}^{+}\left(\mathbf{S}^{n}\right)$.

Fix a cover of $\mathbf{S}^{n}$ by disks $E_{1}, E_{2}$ as in Lemma 6.10. Let $n_{i} \rightarrow \infty$ grow sufficiently quickly, and relabel the sequence

$$
h_{1}^{n_{1}}, h_{1}^{n_{2}}, h_{2}^{n_{2}}, h_{1}^{n_{3}}, h_{2}^{n_{3}}, h_{3}^{n_{3}}, h_{1}^{n_{4}}, \ldots, h_{1}^{n_{i}}, \ldots, h_{i}^{n_{i}}, h_{1}^{n_{i+1}} \ldots
$$

as $H_{0}, H_{1}, H_{2}, \ldots$.

Applying Lemma 6.10, we write each $H_{i}$ as a product

$$
H_{i}=H_{i, 1} H_{i, 2} \ldots H_{i, 6}
$$


where each $H_{i, j}$ has support contained in either $E_{1}$ or $E_{2}$.

From now on, the construction proceeds as in Section 4 and Section 5, with the added simplification that we do not need to worry about the analytic quality of the construction.

We let $D_{i, j}$ be a family of disjoint balls in $\mathbf{S}^{n}$ for $i, j \in \mathbb{Z}$ such that there are homeomorphisms $T, F$ for which $T$ takes $D_{i, j}$ to $D_{i+1, j}$ for all $i, j$, and $F$ takes $D_{0, j}$ to $D_{0, j+1}$, and is the identity on $D_{i, j}$ when $i \neq 0$.

Let $Z_{1}, Z_{2}$ be homeomorphisms taking $D_{0,0}$ to $E_{1}$ and $E_{2}$ respectively.

For each $\ell \in\{1, \ldots, 6\}$ we define $f_{\ell}$ with support contained in the closure of the union of the $D_{i, j}$ by the formula

$$
f_{\ell}=\prod_{i=0}^{\infty} \prod_{j=0}^{\infty} H_{i, \ell}^{Z_{k} F^{-j} T^{-i}}
$$

where $k=1$ if $H_{i, \ell}$ has support in $E_{1}$, and $k=2$ if $H_{i, \ell}$ has support in $E_{2}$.

Then as before, we can write $H_{i, \ell}$ as a word of length $\sim 4 i$ in $f_{1}, \ldots, f_{6}, T, F, Z_{1}, Z_{2}$ and their inverses. In detail:

$$
H_{i, \ell}=Z_{k} y_{i, \ell} Z_{k}^{-1}
$$

where

$$
y_{i, \ell}=w_{i, \ell} F w_{i, \ell}^{-1} F^{-1}
$$

and

$$
w_{i, \ell}=T^{-i} f_{\ell} T^{i}
$$

Since we can do this for each $i, \ell$, we can exhibit each $h_{i}$ as a distortion element, whose distortion function grows as fast as desired. Note that by choosing the $n_{i}$ to all be mutually coprime, we can ensure that the $h_{i}$ are all actually contained in the group in question. 


\title{
Appendix A Strong boundedness of $\operatorname{Homeo}\left(S^{n}\right)$
}

\author{
YVES DE CORNULIER
}

Definition A.1 A group $G$ is strongly bounded ${ }^{1}$ if it satisfies one of the following equivalent conditions:

(i) Every length function on $G$, ie function $L: G \rightarrow \mathbb{R}_{+}$satisfying $L(1)=0$, $L\left(g^{-1}\right)=L(g)$ and $L(g h) \leq L(g)+L(h)$ for all $g, h \in G$, is bounded.

(ii) Every action of $G$ by isometries on a metric space has bounded orbits.

(iii) - $G$ is Cayley bounded: for every symmetric generating subset $S$ of $G$, there exists $n$ such that $G \subset S^{n}=\left\{s_{1} \ldots s_{n} \mid s_{1}, \ldots, s_{n} \in S\right\}$, and

- $G$ has uncountable cofinality, ie $G$ cannot be expressed as the union of an increasing sequence of proper subgroups.

The definition of groups with uncountable cofinality appeared in the characterization by Serre [23, Section 6.1] of groups with Property (FA), meaning that every isometric action on a simplicial tree has a fixed point. For instance, a countable group has uncountable cofinality if and only if it is finitely generated.

Much later, the concept of strong boundedness was introduced by Bergman [1], where it is proved that the permutation group of any set is strongly bounded. Subsequently, intensive research on the subject has been carried on (see, among others, de Cornulier[2], Droste-Holland [3], Kechris-Rosendal [9] and Khelif [10], and the references in Bergman [1]). It is worth noting that a countable group is strongly bounded if and only if it is finite, so that the definition is of interest only for uncountable groups. In Definition A.1, the equivalence between (i) and (ii) is easy and standard; the equivalence between Conditions (i) and (iii) is established in [2] but already apparent in [1].

Fix an integer $n \geq 1$. The purpose of this appendix is to point out the following consequence of the proof of Theorem $\mathrm{C}$ in the paper above.

Theorem A.2 The group Homeo( $\left.\mathbf{S}^{n}\right)$ is strongly bounded.

A weaker version of Theorem A.2 was recently proved by Rosendal [22, Theorem 1.7]; namely, Homeo $\left(\mathbf{S}^{n}\right)$ is strongly bounded as a topological group (for the uniform convergence); this means that every continuous length function is bounded. In [22,

\footnotetext{
${ }^{1}$ The following terminologies for the same concept also exist in the literature: $G$ has the Bergman Property; $G$ has the strong Bergman Property; $G$ has uncountable strong cofinality.
} 
Theorem 5.4], it was also proved that Homeo $\left(\mathbf{S}^{1}\right)$ is strongly bounded. In contrast, if $M$ is any compact non-discrete manifold, then for every $r \geq 1$, the groups $\operatorname{Diff}^{r}(M)$ and their connected components are not strongly bounded. Indeed, the length function $h \mapsto\|d h\|^{+}$defined in Section 3.2 is unbounded.

Proof Clearly, it suffices to show that the subgroup of index two $\mathrm{Homeo}^{+}\left(\mathbf{S}^{n}\right)$ is strongly bounded. By contradiction, we suppose the existence of an unbounded length function $L$ on $G$. Let us pick a sequence $\left(h_{i}\right)$ in $G$ satisfying $L\left(h_{i}\right) \geq i^{2}$ for all $i$.

Using the notation in the proof of Theorem C, set $S=\left\{f_{1}, \ldots, f_{6}, T, F, Z_{1}, Z_{2}\right\}$. Then each $h_{i}$ can be expressed by a word of length $\sim 24 i$ in $S^{ \pm}$. But, on the subgroup of $\mathrm{Homeo}^{+}\left(\mathbf{S}^{n}\right)$ generated by the finite set $S$, the length function $L$ must be dominated by the word length with respect to $S$. This contradicts the assumption $L\left(h_{i}\right) \geq i^{2}$ for all $i$.

Remark A.3 A similar argument to that of the proof of Theorem A.2 was used in [2] to prove that $\omega_{1}$-existentially closed groups are strongly bounded. This reasoning is made systematic by Khelif [10]. Let us say that a group is strongly distorted (introduced as "Property P" in [10]) if there exists an integer $m$ and an integer-valued sequence $\left(w_{n}\right)$ with the following property: for every sequence $\left(h_{n}\right)$ in $G$, there exist $g_{1}, \ldots, g_{m} \in G$ such that, for every $n$, one can express $h_{n}$ as an element of length $w_{n}$ in the $g_{i}$ 's. Following the proof of Theorem A.2, we get that a strongly distorted group is strongly bounded, and that $\operatorname{Homeo}\left(\mathbf{S}^{n}\right)$ is strongly distorted.

The symmetric group on any set is strongly distorted: a proof can be found in Galvin [5], although a weaker result is stated there. In [10], it is claimed that the automorphism group of any 2-transitive chain is strongly distorted; strong boundedness was previously proved by Droste and Holland [3]. On the other hand, if $F$ is a nontrivial finite perfect group, then the infinite (unrestricted) direct product $F^{\mathbb{N}}$ is strongly bounded [2]; however it is clearly not strongly distorted since it is infinite and locally finite.

Remark A.4 It follows from Theorem A.2 that Homeo $\left(\mathbf{S}^{n}\right)$ is Cayley bounded (see Definition A.1); that is, the Cayley graph with respect to any generating subset has bounded diameter. It is natural to ask whether there is a uniform bound on those diameters: the answer is negative. Indeed, endow $\mathbf{S}^{n}$ with its Euclidean metric, and, for $r>0$, set

$$
W_{r}=\left\{g \in \operatorname{Homeo}\left(\mathbf{S}^{n}\right) \mid \forall x \in \mathbf{S}^{n}, d(x, g(x))<r\right\}
$$

and

$$
W_{r}^{+}=W_{r} \cap \text { Homeo }^{+}\left(\mathbf{S}^{n}\right) .
$$


Then $W_{r}^{+}$is open in Homeo ${ }^{+}\left(\mathbf{S}^{n}\right)$. The group Homeo ${ }^{+}\left(\mathbf{S}^{n}\right)$, endowed with the topology of uniform convergence, is connected: this is well-known, and can be deduced, for instance, from Lemma 6.9 above. It follows that $W_{r}^{+}$generates $\mathrm{Homeo}^{+}\left(\mathbf{S}^{n}\right)$. Clearly, for every $k \geq 1$, we have $\left(W_{r}^{+}\right)^{k} \subset W_{k r}^{+}$. It follows that if we have chosen $r \leq 2 / k$, then $\left(W_{r}^{+}\right)^{k} \neq \mathrm{Homeo}^{+}\left(\mathbf{S}^{n}\right)$. Thus Homeo ${ }^{+}\left(\mathbf{S}^{n}\right)$ has Cayley graphs of arbitrary large diameter. A similar argument works for $\operatorname{Homeo}\left(\mathbf{S}^{n}\right)$ as follows: fix a reflection $T \in \mathrm{O}(n+1)$ of $\mathbf{S}^{n}$, and take $W_{r}^{\prime}=W_{r} \cup T W_{r}$. Then it is easy to check that $\left(W_{r}^{\prime}\right)^{k} \subset W_{k r}^{\prime}$ for all $k \geq 1$, so that $\left(W_{r}^{\prime}\right)^{k} \neq \operatorname{Homeo}\left(\mathbf{S}^{n}\right)$ if we have chosen $r \leq 2 / k$.

\section{References}

[1] G M Bergman, Generating infinite symmetric groups, Bull. London Math. Soc. (to appear) arXiv:math.GR/0401304

[2] Y de Cornulier, Strongly bounded groups and infinite powers of finite groups, Comm. Algebra (to appear) arXiv:math.GR/0411466

[3] M Droste, W C Holland, Generating automorphism groups of chains, Forum Math. 17 (2005) 699-710 MR2154425

[4] J Franks, M Handel, Distortion elements in group actions on surfaces arXiv: math.DS/0404532

[5] F Galvin, Generating countable sets of permutations, J. London Math. Soc. (2) 51 (1995) 230-242 MR1325568

[6] J-M Gambaudo, É Ghys, Commutators and diffeomorphisms of surfaces, Ergodic Theory Dynam. Systems 24 (2004) 1591-1617 MR2104597

[7] M Gromov, Hyperbolic groups, from: "Essays in group theory", Math. Sci. Res. Inst. Publ. 8, Springer, New York (1987) 75-263 MR919829

[8] M Gromov, Asymptotic invariants of infinite groups, from: "Geometric group theory, Vol. 2 (Sussex, 1991)", London Math. Soc. Lecture Note Ser. 182, Cambridge Univ. Press, Cambridge (1993) 1-295 MR1253544

[9] A Kechris, C Rosendal, Turbulence, amalgamation and generic automorphisms of homogeneous structures arXiv:math.LO/0409567

[10] A Khelif, À propos de la propriété de Bergman, preprint

[11] RC Kirby, LC Siebenmann, Foundational essays on topological manifolds, smoothings, and triangulations, Princeton University Press, Princeton, N.J. (1977) MR0645390

[12] N Kopell, Commuting diffeomorphisms, from: "Global Analysis (Proc. Sympos. Pure Math., Vol. XIV, Berkeley, Calif., 1968)”, Amer. Math. Soc., Providence, R.I. (1970) 165-184 MR0270396 
[13] S Lang, Algebra, Graduate Texts in Mathematics 211, Springer, New York (2002) MR1878556

[14] A Lubotzky, S Mozes, MS Raghunathan, The word and Riemannian metrics on lattices of semisimple groups, Inst. Hautes Études Sci. Publ. Math. (2000) 5-53 (2001) MR1828742

[15] G D Mostow, Strong rigidity of locally symmetric spaces, Princeton University Press, Princeton, N.J. (1973) MR0385004

[16] A Y Ol'shanskii, Distortion functions for subgroups, from: "Geometric group theory down under (Canberra, 1996)", de Gruyter, Berlin (1999) 281-291 MR1714850

[17] D Pixton, Nonsmoothable, unstable group actions, Trans. Amer. Math. Soc. 229 (1977) 259-268 MR0438397

[18] M Pollicott, Lectures on ergodic theory and Pesin theory on compact manifolds, London Mathematical Society Lecture Note Series 180, Cambridge University Press, Cambridge (1993) MR1215938

[19] L Polterovich, Growth of maps, distortion in groups and symplectic geometry, Invent. Math. 150 (2002) 655-686 MR1946555

[20] F Quinn, Ends of maps III: Dimensions 4 and 5, J. Differential Geom. 17 (1982) 503-521 MR679069

[21] D Repovš, E Ščepin, A proof of the Hilbert-Smith conjecture for actions by Lipschitz maps, Math. Ann. 308 (1997) 361-364 MR1464908

[22] C Rosendal, A topological version of the Bergman Property arXiv: math.LO/0509670

[23] J-P Serre, Arbres, amalgames, $\mathrm{SL}_{2}$, Société Mathématique de France, Paris (1977) MR0476875

[24] Y G Sină, Topics in ergodic theory, Princeton Mathematical Series 44, Princeton University Press, Princeton, NJ (1994) MR1258087

[25] T Tsuboi, Homological and dynamical study on certain groups of Lipschitz homeomorphisms of the circle, J. Math. Soc. Japan 47 (1995) 1-30 MR1304186

Department of Mathematics, California Institute of Technology, Pasadena CA 91125, USA Microsoft Research, 1 Microsoft Way, Redmond WA 98052, USA

Yves de Cornulier: Institut de Mathématiques, Université de Neuchâtel Rue Émile Argand 11, CH-2007 Neuchâtel, Switzerland dannyc@its.caltech.edu, michaelf@microsoft.com, yves.cornulier@ens.fr

Proposed: Benson Farb

Seconded: Leonid Polterovich, Robion Kirby
Received: 7 October 2005

Revised: 20 February 2006 